\title{
Statistical comparison of transition region blinkers and explosive events
}

\begin{abstract}
A. Brković and H. Peter
Kiepenheuer-Institut für Sonnenphysik, 79104 Freiburg, Germany

e-mail: alen@kis.uni-freiburg.de

Received 19 March 2004 / Accepted 19 April 2004

Abstract. Explosive events and blinkers are two observational classes of transients seen on the quiet Sun and an investigation of the significance of and relationship between such events may be critical for understanding basic processes at work in the solar atmosphere. We analysed five time-series spectra of the quiet Sun of transition region Ov $629 \AA$, O vi $1032 \AA$ and O vi $1038 \AA$ lines. We investigated how often explosive events occurred during the course of a blinker at the same location and found that slightly more than a half of all explosive events happened during about one third of all blinkers. In some cases during a blinker more than one explosive event was registered. The largest average maximum relative intensity enhancement was for blinkers with explosive events, followed by the blinkers without explosive events, with the least being for the explosive events which did not happen during the course of a blinker. Due to these differences among the maximum enhancements between these events we suppose that blinkers and explosive events are two independent phenomena. Intensity light curves of blinkers show that events can be separated into two classes, specifically: 1) simple blinkers with smooth increase in intensity having only one significant peak, and 2) complex blinkers characterised by multiple (2-4) significant peaks. These two classes were equally represented when frequencies of their occurrences were averaged over five analysed data-sets. The analysis of the line profile parameters and their correlations for these two classes did not give any result which could further distinguish between them. During blinkers the intensity peaks mostly at the middle of their durations, while the line width peaks somewhat earlier than the intensity. This was a general character for both simple and complex blinkers, as well as for all explosive events.
\end{abstract}

Key words. Sun: chromosphere - Sun: corona - Sun: transition region - Sun: UV radiation

\section{Introduction}

Two observational classes of transients seen on the quiet Sun are explosive events and blinkers. If and how such events are related one to another still needs to be investigated. A such study is needed because it may contribute to our understanding of basic processes at work in the solar atmosphere. Explosive events are transition region phenomena first reported by Brueckner \& Bartoe (1983) and Dere et al. (1984). The time scale of these short living events is about one minute and their blue and red components were shifted on average by some $100 \mathrm{~km} \mathrm{~s}^{-1}$. The explosive events were not necessarily related to a strong brightening. Observational studies performed using the Solar Ultraviolet Measurements of Emitted Radiation (SUMER; Wilhelm et al. 1995) specrometer on SOHO clarified that explosive events are bi-directional jets following a reconnection event (Innes et al. 1997; Innes \& Tóth 1999).

To test if explosive events occur basically in the transition region the search for their coronal signature has been undertaken, as well. Analysing the $\mathrm{Fe}$ Ix/Fe $\mathrm{x} 171 \AA\left(10^{6} \mathrm{~K}\right) \mathrm{TRACE}$ (Transition Region and Coronal Explorer, Handy et al. 1999) images of an active region together with SUMER C IV $1548 \AA$ $\left(10^{5} \mathrm{~K}\right)$ rasters, Winebarger et al. (2002) found a modest signature of explosive events in the corona. Their argument was that this result could also be due to the transition region $\mathrm{O}$ VI $172 \AA\left(3 \times 10^{5} \mathrm{~K}\right)$ line within the TRACE Fe Ix/Fe x 171 passband. Teriaca et al. (2002) also searched for the coronal signature of explosive events. They used the SUMER instrument to observe simultaneously the coronal Mgx line $624 \AA\left(10^{6} \mathrm{~K}\right)$ and the transition region line $\mathrm{Nv} 1238 \AA\left(1.8 \times 10^{5} \mathrm{~K}\right)$. The analysis of line profiles and integrated intensities of the $\mathrm{Mg} x$ line showed the absence of any signs of explosive events. On the other hand, they found the response of the low transition region observed in the $\mathrm{S}_{\text {II }}$ line $1253 \AA\left(3.5 \times 10^{4} \mathrm{~K}\right)$ to explosive events in Nv. Madjarska \& Doyle (2002) also used SUMER and found that the emission in H I Ly $6^{1} 930 \AA$ is correlated to explosive events detected in S VI $933 \AA\left(1.9 \times 10^{5} \mathrm{~K}\right)$.

Another transition region phenomenon is EUV brightenings, so-called blinkers, first reported by Harrison (1997). The author analysed quiet-Sun observations obtained by the Coronal Diagnostic Spectrometer (CDS; Harrison et al. 1995) on SOHO. He found that blinkers are best observed in the transition region lines of $\mathrm{O}_{\text {III }} 599 \AA\left(10^{5} \mathrm{~K}\right)$, O IV $554 \AA$

1 According to Fontenla et al. (1993) H H Ly 6 is formed in the uppermost part of the chromosphere above $9000 \mathrm{~K}$. 
$\left(1.6 \times 10^{5} \mathrm{~K}\right)$ and $\mathrm{O} v 629 \AA\left(2.5 \times 10^{5} \mathrm{~K}\right)$. The main characteristic of these events was an intensity increase of typically $60-80 \%$, and duration of 5 to $30 \mathrm{~min}$. These studies showed that blinkers are mainly located in the network. Together with the transition region lines Harrison et al. (1999) simultaneously observed the chromospheric $\mathrm{He}_{\mathrm{I}} 584 \AA$ Alne $\left(2 \times 10^{4} \mathrm{~K}\right)$ and the coronal Mg IX $368 \AA\left(10^{6} \mathrm{~K}\right)$ and $\mathrm{Mg}$ x $624 \AA$ A lines. Significant increases in brightness in chromospheric and coronal lines have not been reported in the study. Further reports on quiet-Sun blinkers observed with CDS are those of, e.g., Brković et al. (2001), Bewsher et al. (2002). Brković et al. (2001) detected blinkers both in the network and the inter-network. Bewsher et al. (2002) reported that blinkers are approximately located above regions of strong unipolar field. Parnell et al. (2002) studied blinkers in active regions and found very similar results to the quiet-Sun blinker studies. The difference is reflected through larger enhancements and frequency of occurrence in active regions. Blinkers were also detected in the upper (O VI $1038 \AA, 3 \times 10^{5} \mathrm{~K}$ ) and lower (C II $1037 \AA, 2-4 \times 10^{4} \mathrm{~K}$ ) transition region lines using SUMER (Brković \& Peter 2003). Enhancement levels for $\mathrm{C}_{\mathrm{II}}$ and $\mathrm{O}$ VI blinkers were similar and on average above $100 \%$. Brković \& Peter (2003) found that even the low chromosphere shows signatures of blinkers. They found blinkers to be accompanied by enhancements in the C I continuum (near $1040 \AA$ ) formed just above the temperature minimum.

While explosive events are widely thought to be due to reconnection events, the cause for blinkers is yet to be determined. Since blinkers are defined as brightenings that exceed a certain threshold, we can imagine that several mechanisms can lead to the significant excess in the intensity, that can be detected as a blinker. Several suggestions and models were presented which try to explain blinkers. Harrison (1997) and Harrison et al. (1999) inspected ratios of transition region lines and found that these ratios show small variations in time during a blinker. On the basis of this result they concluded that blinkers are probably caused by density enhancements or changes in brightness filling factor, and are not due to a temperature effect. However, the contribution functions of the three transition region lines are quite broad on the temperature scale and overlap each other (Arnaud \& Rothenflug 1985). Due to this we should not exclude changes in the plasma temperature as a possible cause for blinkers. A further blinker mechanism based on observations is given by Peter \& Brković (2003). Studying the time evolution of the intensity, line width and Doppler shift, they concluded that blinkers are driven by heating a loop asymmetrically and powering a strong laminar flow through the loop. Several other theoretical scenarios for the origin of blinkers were presented by Priest et al. (2002). In one of these processes blinkers might be the consequence of exploding granules in the chromosphere. Numerical 2D simulations by Marik \& Erdélyi (2002) treated the blinker in the same way as an explosive event. The authors argued that blinkers are in fact explosive events, and the lack of velocities detected in blinkers by CDS is due to the relative spatial resolutions of the CDS and SUMER instrument. These simulations covered six minutes which is on the lower end of the distribution of blinker durations.
Although observational characteristics of blinkers and explosive events are different it is worth to establish the significance of and confirm relationship between them. This might be of importance for understanding basic processes occurring in the solar atmosphere. Existing observational results are not consistent. Studies which suggest a close relationship between the blinkers and explosive events are those of Chae et al. (2000) and Innes (2001), both papers discussed by Harrison et al. (2003). In the work of Chae et al. (2000) simultaneous observations of explosive events obtained with SUMER and of blinkers detected using CDS were compared. The authors showed that explosive events tend to occur on the edges of and simultaneous with blinkers. Similarly, Innes (2001) compared SUMER observations to EUV images from TRACE and confirmed that the $\mathrm{C}$ IV transition region line from TRACE exhibits brightenings in association with velocity events. Contrary to these findings Madjarska \& Doyle (2002) found no signs of explosive events detected with SUMER at the blinker locations determined with CDS. Though, the six explosive events they detected harboured intensity increases typical for blinkers. The work of Peter \& Brković (2003) also showed that in the particular blinker-case they analysed no signs of explosive events were present. They proposed the existence of a class of blinkers that does not share spectral characteristics of explosive events.

The relationship between explosive events and blinkers still needs further investigation but this work contributes significantly to our understanding of these events. In our approach we use the SUMER instrument to investigate the spectral profiles, intensity, line width and Doppler shift light curves of transition region lines O v $629 \AA$, O vi $1032 \AA$ and O vi $1038 \AA$.

\section{Observations}

To detect both explosive events and blinkers with high spatial resolution and to estimate accurate velocities, we use the SUMER instrument aboard SOHO. Its spatial resolution is $1.2^{\prime \prime} \times 2^{\prime \prime}$ for the $0.3^{\prime \prime} \times 120^{\prime \prime}$ slit (Lemaire et al. 1997) and it can reliably measure velocities as low as $2 \mathrm{~km} \mathrm{~s}^{-1}$ (Peter \& Judge 1999). These capabilities are of great advantage if we want to detect traces of explosive events and blinkers at the same location and time. Using only one instrument we avoided the problems (different spatial, spectral and temporal resolutions, different fields of view and co-pointing) arising when two, or more, instruments are involved in simultaneous and co-spatial observations. Here we present the analysis of SUMER data of SOHO Joint Observing Program (JOP) 61 and JOP 72. Dates of observations, lines, exposure times and slits used are given in Table 1. Quiet-Sun regions near the disc centre were observed with SUMER's detector B and the slit was kept at a fixed location on the solar surface by compensating for solar rotation. Due to misalignment of slit and detector not all data are of use (70-90\%).

Instrumental corrections that we applied account for: flatfield, pin-cushion distortion of the image introduced by the detector, the inclination of the spectral lines with respect to the detector columns, dead time and gain depression of the detector. Once we performed these established SUMER procedures, in order to increase the signal-to-noise ratio of the spectra we 

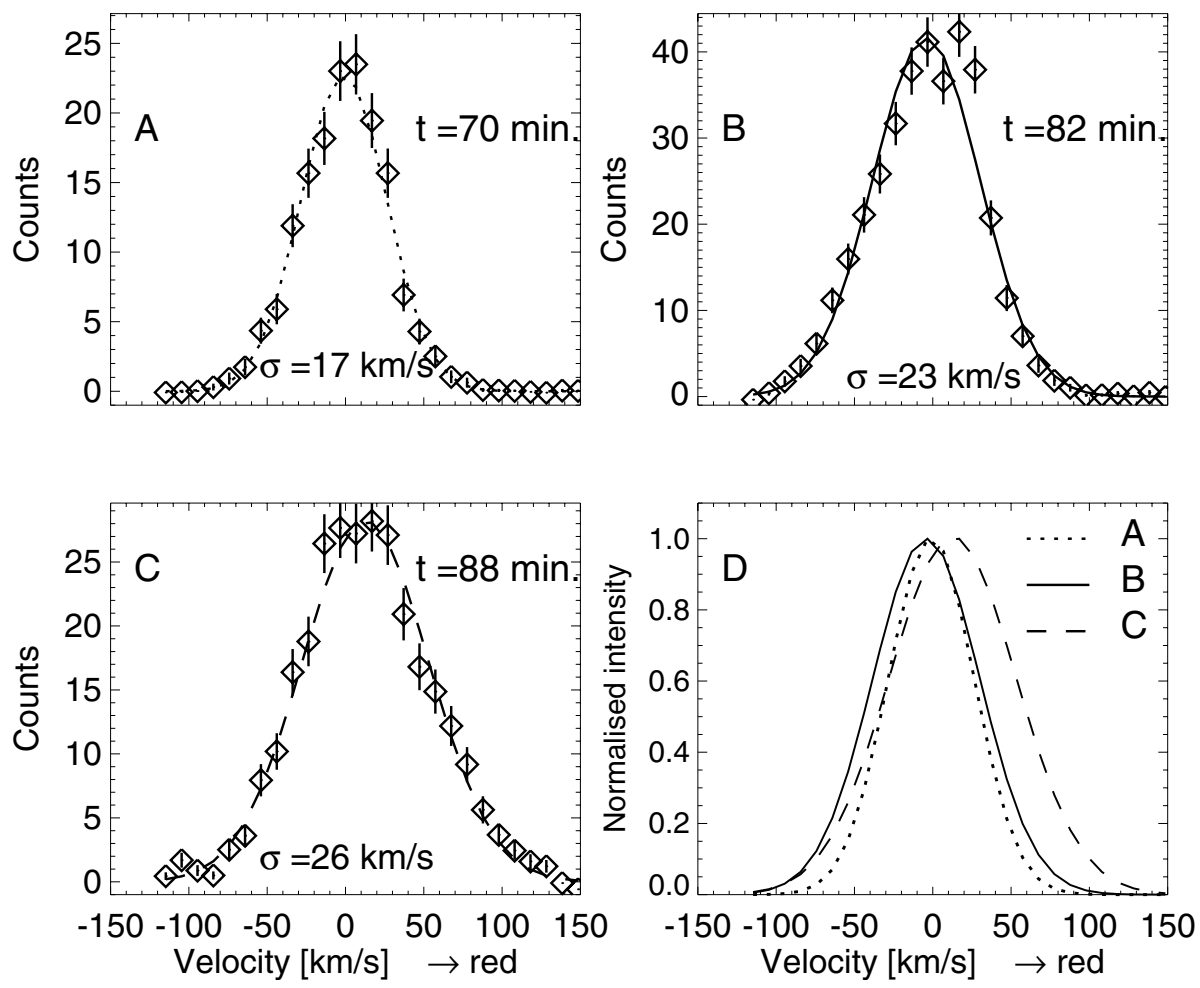

Fig. 1. Three sample spectra (A, B and C) from Fig. 2 shown as bars that represent the Poisson errors. The single Gaussian fits are shown as lines. The A spectrum has less broadened line profiles than the B and $\mathrm{C}$ spectra. The line widths $(\sigma)$ are also given on the plots. For a better comparison of the line widths, panel D shows only Gaussian fits with normalised intensities.

Table 1. Observational details. Width refers to the width of the spectral window (see Sect. 2).

\begin{tabular}{cccccccc}
\hline \hline Date & $\begin{array}{c}\text { Start-End } \\
\text { Time (UT) }\end{array}$ & $\begin{array}{c}\text { Exposure time } \\
\text { + Overhead (s) }\end{array}$ & $\begin{array}{c}\text { Slit No. } \\
\left(\operatorname{arcsec}^{2}\right)\end{array}$ & $\begin{array}{c}\text { Final FOV along } \\
\text { the Slit }(\operatorname{arcsec})\end{array}$ & $\begin{array}{c}\text { Line } \\
(\AA)\end{array}$ & $\begin{array}{c}\log \left(T_{\mathrm{e}} / \mathrm{K}\right) \\
(\AA)\end{array}$ & $\begin{array}{c}\text { Width } \\
(\AA)\end{array}$ \\
\hline $97-04-22$ & $09: 35-13: 47$ & $15+4$ & $6(0.3 \times 120)$ & 85 & O vi 1032 & 5.50 & 2.2 \\
$97-04-25$ & $12: 46-18: 19$ & $15+4$ & $6(0.3 \times 120)$ & 85 & O vi 1032 & 5.50 & 2.2 \\
$98-05-12$ & $14: 36-16: 02$ & $10+2$ & $8(0.3 \times 120)$ & 96 & O vi 1038 & 5.50 & 2.2 \\
$98-05-16$ & $14: 30-16: 40$ & $10+2$ & $2(1.0 \times 300)$ & 270 & O vi 1038 & 5.50 & 2.2 \\
$97-04-23$ & $13: 44-18: 43$ & $30+4$ & $3(1.0 \times 120)$ & 89 & O v 629 & 5.39 & 1.1 \\
\hline
\end{tabular}

applied running means in time with a box width corresponding to $150 \mathrm{~s}$. The width of the box is chosen as a compromise between the goodness of the fit $\chi_{r}^{2}$ and the time resolution.

Spectral line data were further fitted with either single Gauss fits (April 1997 data) or double Gauss fits (May 1998 data). Double Gauss fits were needed in order to separate the C II $1037 \AA$ and O VI $1038 \AA$ lines. Least-squares fits were applied and a linear background was subtracted in the fits. The fitting procedure used a Genetic Algorithm based optimisation method of Charbonneau (1995). This procedure, fitting single Gaussians to the line profile, will not well represent clear explosive events with distinct satellite components. Nevertheless, using single Gaussian fits these events will then be clearly visible in the line width (cf., Fig. 1). The line width during an explosive event should thus be considered as a measure for the strength of the explosive event.

Since the absolute calibration of wavelengths was not necessary for this study we did not perform it. Line of sight velocities are given relative to the position of the line obtained after averaging over all spatial pixels and all time steps for a particular data set.

\section{Event identification}

\subsection{Blinker identification}

To identify blinkers in $\mathrm{Ov}$ and $\mathrm{O} v \mathrm{v}$ we used the same automated routine as applied by Brković et al. (2001). Figure 2 shows a portion of the light curve of a pixel which we use to describe the four steps of our method of identifying blinkers. Step 1: for each pixel we first determine the time steps at which the intensity is above a given threshold that is chosen to be some factor times the time-averaged intensity of that pixel (solid horizontal line in Fig. 2). Step 2: starting from the local maximum lying above the threshold we follow the intensity both backward and forward in time, until local minima are found which lie below the time-averaged intensity of the pixel in question (termed the background intensity; $I_{\text {back }}$ ). These points are designated as the preliminary start and end times of a brightening event. Step 3: if one or more brightness events are identified in a particular pixel a new background intensity is calculated by averaging the intensities of only those time steps not harbouring such events. Steps 1-3 are then repeated with the new (lower) background intensity until no new event is identified. This is the case after less than five 

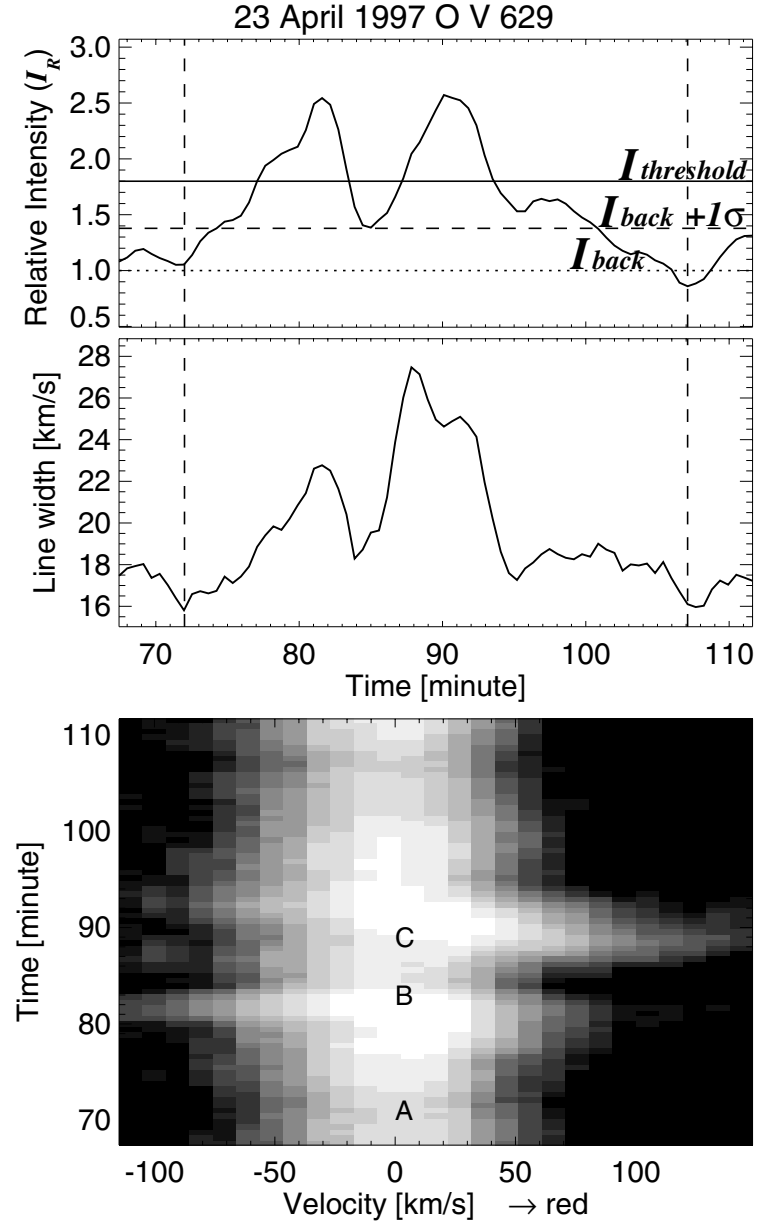

Fig. 2. Example of a blinker and two explosive events detected during the blinker at the same location. Start and end times of the blinker are 72 and 107 min. Two explosive events took place around 82 (B) and 88 (C) min. This is a $45 \mathrm{~min}$ part of five hours time series.

iterations for almost all spatial pixels in our data sets. From now on all intensities are given relative to this final background intensity and are designated as $I_{\mathrm{R}}$ in Fig. 2. Finally, in Step 4 we determine the standard deviation $(\sigma)$ of the intensity at the time-steps not harbouring brightness events (i.e. of the background intensity) and we repeat Step 2, but this time looking for local minima below the final background intensity plus the $1 \sigma$ standard deviation above this value (horizontal dashed line in Fig. 2). This further step was introduced since in a number of cases brightness events with extremely long lifetimes were found, which only had a single relatively short-lived peak lying above the threshold, however. Since in most such cases the intensity just fails to drop below the background relatively close to the peak we decided that these long lifetimes are often an artifact of noise or intrinsic, weak background variability, and introduced Step 4.

Since some brightness events started before the beginning or ended after the end of our observations their starting and ending times cannot be determined and consequently they are not retained for further analysis.
Table 2. Distribution of blinkers and explosive events. An example of the respective events can be found with the figure number (\#).

\begin{tabular}{lccc}
\hline \hline & Fig. \# & & \\
\hline Number of blinkers & & 1086 & \\
- Blinkers with expl. events & 2 & 342 & $(32 \%)$ \\
- Simple blinkers & 4 & 560 & $(52 \%)$ \\
- Complex blinkers & 5 & 526 & $(48 \%)$ \\
\hline Number of explosive events & & 778 & \\
- Expl. events during blinkers & 2 & 412 & $(53 \%)$ \\
- “Classical” expl. events & 3 & 366 & $(47 \%)$ \\
\hline
\end{tabular}

\subsection{Explosive event identification}

The identification of explosive events was performed by eye inspection of velocity-time plots of line profiles for each pixel. The cases with "strong" satellite components (velocity larger than $50 \mathrm{~km} \mathrm{~s}^{-1}$ towards the blue or red) we registered as explosive events. The limit of $50 \mathrm{~km} \mathrm{~s}^{-1}$ is not set as the only condition for assigning explosive events. Not each case with velocities greater than this limit is determined as an explosive event. Another important condition is the shape of the velocitytime plot. This method is illustrated in Fig. 2 which shows two explosive events taking place at 82 and $88 \mathrm{~min}$. The events were recorded in the Ov line on 23 April 1997. We allow that a certain degree of subjectivity can not be avoided in this detection method. Since explosive events live much shorter than blinkers, for the determination of their start and end times we included two minutes before and after the actual event in their life-times. This was necessary in order to have enough time steps used for further analysis described in the next section.

\section{Results}

The intention of this study was the investigation of the relationship between explosive events and blinkers and the comparison between simple and complex blinkers. The identification methods detected 1086 blinkers and 778 explosive events in five data-sets (see Table 2). Usually these transient events were visible in two or more neighbouring pixels. In these cases, however, the transients seen in neighbouring pixels were of the same type. For instance, we did not see an explosive event in one pixel and a blinker in the pixel next to it. In this study it was not necessary to spatially group the events, so that each event is analysed as being comprised of a single pixel. "Cotemporality" refers to the common time steps of co-spatial (recorded in the same pixel) blinkers and explosive events. On average, "co-temporal" explosive events spent almost $90 \%$ of their time during a blinker. Without implying any other meaning for such co-spatial and "co-temporal" blinkers we apply the term "blinker with an explosive event".

To show the characteristics of the respective events we display in the sample figures (Figs. 2-5) the evolution of the spectrum in time (velocity-time plot; bottom panel), the light curve (relative intensity vs. time; top panel), and the line width plot 

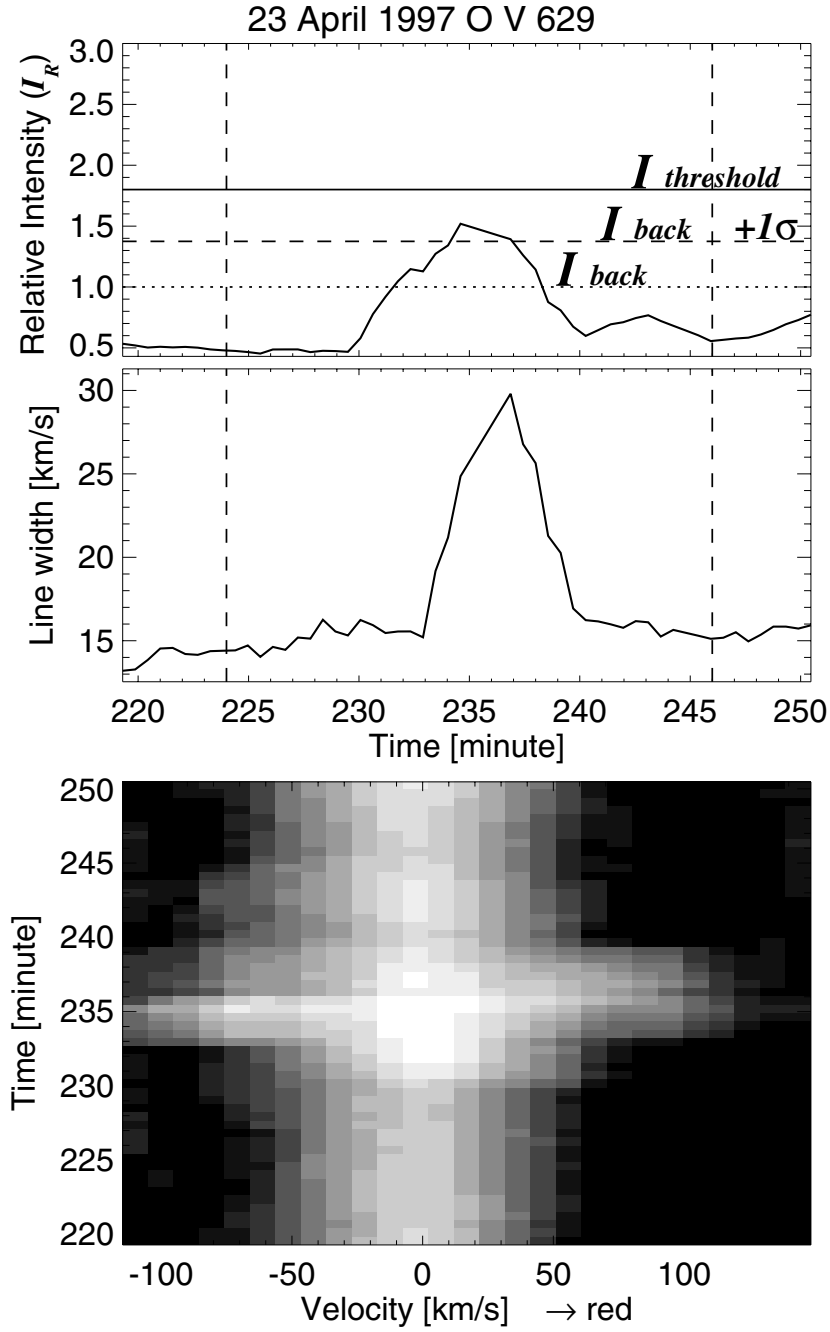

Fig. 3. Example of a "classical" explosive event, i.e. an explosive event without a blinker signature according to our criteria, taking place between 233 and $238 \mathrm{~min}$. This is a $31 \mathrm{~min}$ part of five hours time series.

(line width after correction for instrumental broadening; middle panel).

\section{1. "Classical" explosive events}

Here we report on "classical" explosive events, i.e. on those explosive events which did not happen during the course of a blinker. An example of a "classical" explosive event is given in Fig. 3. The velocity-time plot and line width curve clearly show signatures of explosive event activity between 233 and $238 \mathrm{~min}$. The corresponding intensity light curve shows that no blinker occurred at that time, according to our criteria. The number of "classical" explosive events detected in the data-sets we analysed was 366 , or $47 \%$ of all detected explosive events.

Further, we inspected the behaviour of the line profile parameters during these explosive events. On average, the correlations between the parameters are not significant. In the next step we determined times when the intensity and line width peak relative to the whole duration of the events and call them peak times. Peak times for the intensity and line width are narrowly distributed with overall average values being almost
Table 3. Maximum relative intensities $\left(I_{\mathrm{R}} \max \right)$, peak times of the intensity $(I)$ and line width $(L W)$ for various classes of events. The maximum relative intensity is the maximum intensity during an event given relative to the time-averaged intensity. The peak times are the time-steps when the parameters peak relative to the whole duration of the event. Bl. and bl. mean blinkers.

\begin{tabular}{lccccc}
\hline \hline & $\begin{array}{c}\text { Bl. free } \\
\text { of EE }\end{array}$ & $\begin{array}{c}\text { Bl. } \\
\text { with EE }\end{array}$ & $\begin{array}{c}\text { Simple } \\
\text { bl. }\end{array}$ & $\begin{array}{c}\text { Complex } \\
\text { bl. }\end{array}$ & $\begin{array}{c}\text { Classical } \\
\text { EE }\end{array}$ \\
\hline$I_{\mathrm{R}} \max$ & 2.4 & 3.1 & 2.8 & 2.7 & 1.6 \\
$I$ peak $t$ & 0.49 & 0.50 & 0.46 & 0.51 & 0.47 \\
$L W$ peak $t$ & 0.42 & 0.40 & 0.43 & 0.44 & 0.45 \\
\hline
\end{tabular}

the same, 0.47 and 0.45 . The correlation coefficients between the peak times are small and not significant. Average maximum enhancements of the relative intensities are defined as $\overline{I_{\mathrm{R}}(\max )}-1$, where $\overline{I_{\mathrm{R}}(\max )}$ is the average of maximum relative intensities (Table 3 ). According to this definition average maximum enhancements for explosive events amount to $60 \%(0.6=1.6-1)$.

\subsection{Simple and complex blinkers}

Two time-structured classes of blinkers, simple and complex ones, were reported by Harrison et al. (1999) and Brković et al. (2001). This visually performed classification is based on the shape of the intensity light curve during a blinker. The simple blinker is characterised by having smooth rise in the intensity and one significant peak, while the complex blinker by multiple (2-4) significant peaks. No other particularities of the two classes have been reported so far.

Examples of two simple blinkers are given in Fig. 4. Their start times are at 8 and $23.5 \mathrm{~min}$ and they last 15.5 and $18 \mathrm{~min}$, respectively. The velocity-time plot does not reveal strong excursions in the velocity, and in the line width there are no signs typical for explosive events. On the contrary, the line width drops during the blinker, as reported by Peter \& Brković (2003). Thus, in this particular case no explosive event took place during blinkers. The intensity light curve rises (declines) smoothly and contains only one significant peak during each blinker. Due to such "simple" character of the light curve this class of blinkers was so termed. About a half of all blinkers detected in the five data-sets could be classified as simple blinkers, and during one third of them at least one explosive event took place. The study of line profile parameters during simple blinkers without explosive events gave no new significant results. It is interesting to note the relationship between the intensity and line width as can be seen in Fig. 4. Such behaviour was unequally represented in the five data-sets we analysed, but in the dataset of 16 May 1998 we found it quite often among simple blinkers.

Figure 5 shows an example of a complex blinker starting at 53 and ending at $91.6 \mathrm{~min}$. The corresponding velocity-time plot shows no signatures of explosive event activity in this time interval. Different to the simple-blinker case the intensity light curve contains four significant peaks and therefore the term 


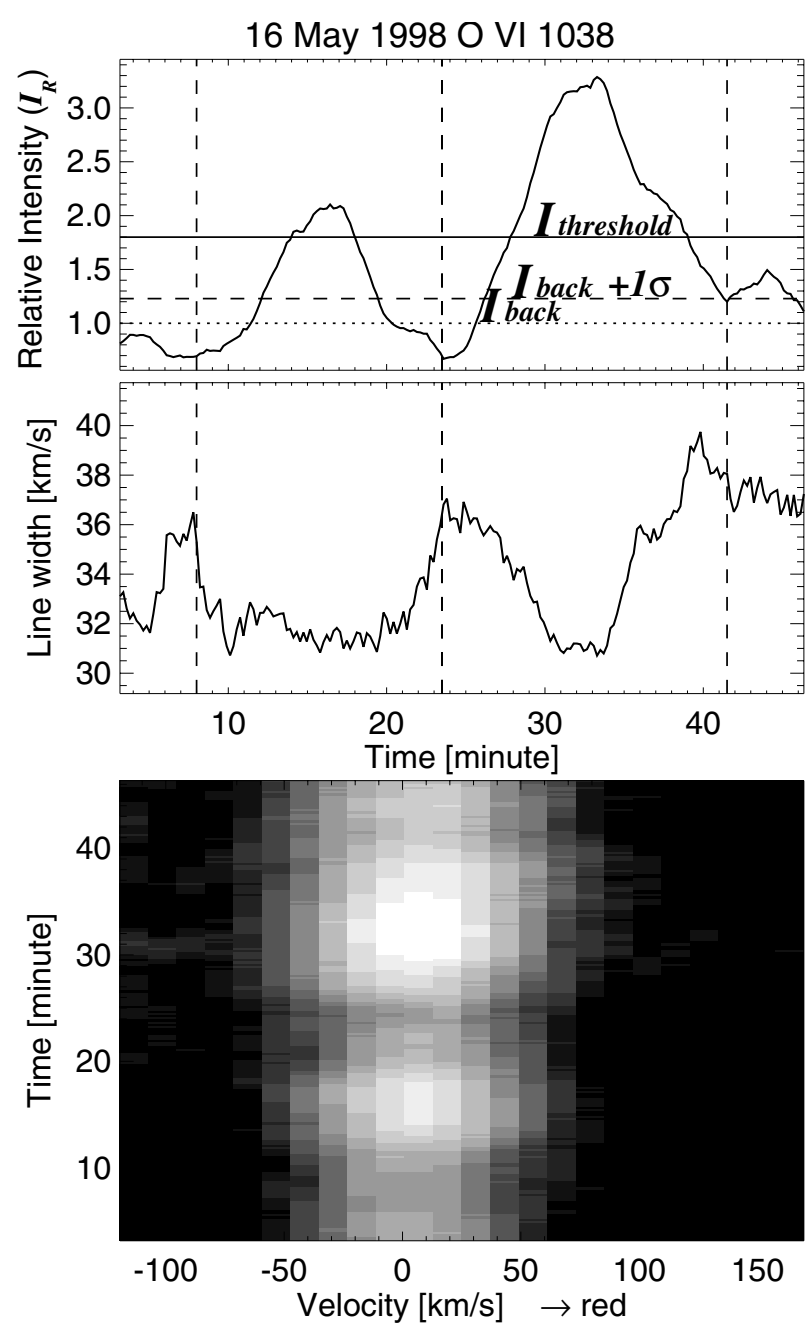

Fig. 4. Examples of two simple blinkers starting at 8 and $23.5 \mathrm{~min}$ and ending at 23.5 and $41.5 \mathrm{~min}$. This is a $43 \mathrm{~min}$ part of two hours and 20 min time series.

"complex" blinker. Blinkers with two or three significant peaks were also detected. The visual inspection of complex blinkers showed that these peaks are separated by between 5 and $10 \mathrm{~min}$ (cf. Fig. 5). During 30\% of complex blinkers at least one explosive event took place. The study of line parameters during complex blinkers without explosive events gave no new significant results, as was the case for simple blinkers. The classification of blinkers into the simple and complex classes is not free of subjectivity. A good example of this subjectivity is given in Fig. 2 where, according to our criteria, we have a complex blinker. But, if the local minima at around 85 min were below the dashed line we would have had two simple blinkers instead. A similar problem in the blinker identification method is already mentioned in Sect. 3.1.

Considering simple and complex blinkers without explosive events we determined the times when the intensity and line width peaks relative to the whole duration of the events. Average values of the intensity peak times are narrowly distributed somewhat below 0.5 , giving an average of 0.48 . The peak times of line widths are distributed around 0.4 with an average of 0.42 . The correlation coefficients between the
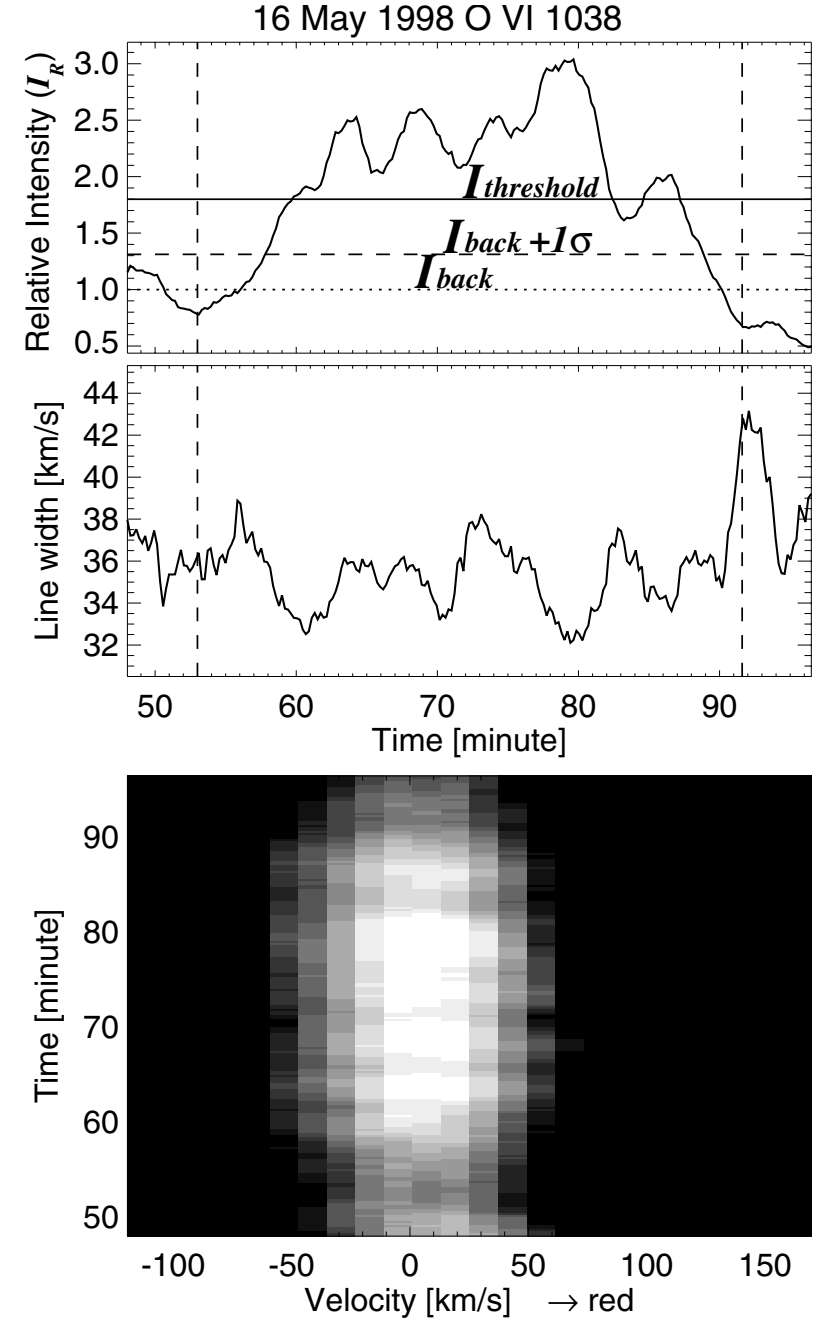

Fig. 5. Example of a complex blinker starting at 53 and ending at $91.6 \mathrm{~min}$. This is a $48 \mathrm{~min}$ part of two hours and $20 \mathrm{~min}$ time series.

intensity and line width peak times in each data-set are small and insignificant.

\subsection{Blinkers-explosive events relationship}

The final step in the study of the relationship between blinkers and explosive events was to compare the locations and times of these events. This was needed in order to determine how often explosive events took place during the course of a blinker. Table 2 shows these occurrence frequencies. In all data-sets we detected 412 such cases. Relative to the total number it means that $53 \%$ of all detected explosive events had intensity increases typical for blinkers. Since blinkers have longer lifetimes than explosive events there were cases in which more than one explosive event was registered during a blinker. The total number of such blinkers is 342 , i.e. $<412$, what is $\approx 32 \%$ of all blinkers. From Table 2 we see that for a given data-set the average number of explosive events during a blinker is about $1.2(=412 / 342)$.

An example of a blinker with an explosive event is shown in Fig. 2. The plot shows about 44 min of the whole time series. The blinker started at $72 \mathrm{~min}$ and ended at $107 \mathrm{~min}$. Two 
explosive events were recorded at around 82 and $88 \mathrm{~min}$. The velocity-time plot clearly reveals the presence of strong blue and red wings, while the line-width light curve shows significant increases in the line width during events. Both are characteristics for explosive events.

To further investigate the character of blinkers with explosive events we inspected the behaviour of the line profile parameters during these events. Average values of the intensity peak times are narrowly distributed around 0.5 , i.e. the middle of the event's duration. On the other hand, the line widths peaked earlier than the intensity, at about 0.4 . These results are similar to the one from Sects. 4.1 and 4.2. Average maximum enhancements for blinkers with explosive events, however, are $210 \%$, four times larger than for the "classical" explosive events. This confirms that explosive events are not necessarily related to strong brightenings.

\section{Discussion}

We first discuss the relationship between explosive events and blinkers based on the correlation study between line profile parameters during these events. The main results of this correlation study are:

- Correlations between the line profile parameters during blinkers and explosive events were not significant. The only trend concerning these correlations was found between the intensity and line width for blinkers with explosive events and for "classical" explosive events.

- The corresponding correlation coefficients were always positive. It seems that this, though small, positive intensityline width correlation was due to explosive events.

- The inspection of times when these two parameters peak showed that they both, on average, peak around the middle of the events. The correlations between these peak times did not reveal any trend, which could mean that the process(es) which cause(s) the intensity and line width peaks do not know each other.

The results concerning values of the maximum relative intensities showed that these values were highest for blinkers with explosive events with an average of 3.1. The average value of this parameter for blinkers without explosive events was 2.4 and for "classical" explosive events only 1.6.

Since these parameters are significantly different for blinkers with and without explosive events we believe that they point to an independent nature of blinkers and explosive events. We speculate that although we detect a blinker and an explosive event at the same location and almost at the same time the two events are actually different processes, blinkers leaving a strong signal in the intensity and explosive events strong signal(s) in the line width and/or line of sight velocity. If both of these events by chance happen in a close time interval along the SUMER's line of sight their emissivities will be added. This "coincidence" can lead to the stronger observed intensity than in the case when only one of these events takes place. This idea is also supported by the results concerning (non-correlated) peak times of the intensity and line width and clarifies the (non)relationship between blinkers and explosive events. We can not rule out the possibility that both events are ultimately two different realizations of some single event-type which can lead to heating and flows. The observations showed that for some events we see flows and no heating and for others we see heating and no flows and finally there are some events in between, but for the single event-type scenario we still miss the evidence.

Peter \& Brković (2003) analysed one particular blinkercase which was not related to explosive events. They analysed the line profiles of the spectra forming a blinker and gave physical reasons for the difference between the two events. Recall that an explosive event leaves its signature mainly through the broadening of the line or in the line wings. The work in the current paper supports their results. Madjarska \& Doyle (2002) compared times and locations of six explosive events and found that they did not coincide with any blinker and concluded that the two events were independent. They also found that during these explosive events the levels of intensity increases were of orders typical for blinkers. On the other hand we found quite a high overlap in locations and times of blinkers and explosive events. Similarly, a quite good agreement in the locations and times of the two events were also reported by Chae et al. (2000) and Innes (2001). Our result does not support the work of Marik \& Erdélyi (2002) who performed 2D numerical simulations of explosive events. They argued that blinkers are in fact explosive events, and the lack of velocities detected in blinkers by CDS is due to the relative spatial resolutions of the CDS and SUMER instrument.

The time-structured, simple and complex, character of blinkers has been reported by Harrison et al. (1999) and Brković et al. (2001). This classification is performed according to the number of significant peaks during a blinker and the shape of its light curve. We searched to see if we could find some other feature(s) which could make this distinction clearer. Neither correlations between the line parameters nor correlations of the times when the intensity and line width peak showed any tendency or significance. So, at least on the basis of these analyses we found no further clue that could help us to answer on this question.

On the basis of the analysis of one simple blinker with informations on the intensity, line width and line of sight velocity, Peter \& Brković (2003) argued that such blinkers are driven by steady asymmetric heating of a coronal loop and powering a strong laminar flow through the loop. The intensity and line width light curves of the case they analysed were very similar to the example of the simple blinker given in Fig. 4. In this work we could not give conclusive evidence favouring their proposal, but we could report on many observational cases for that kind of simple blinkers.

On the other hand some modelling efforts have been undertaken to try to clarify the difference between simple and complex blinkers. Recently, Peter et al. (2003) applied a 1D loop model which includes a proper energy equation and nonequilibrium ionisation balance. In the model the heating was increased by a factor of four in one leg and spectra integrated over the whole loop were calculated. The simulation covered about $15 \mathrm{~min}$ and was able to reproduce a blinker-like curve in the transition region Ov $629 \AA$ line. Peter et al. (2003) also 
tried to numerically simulate complex blinkers. In this case they increased pressure below one leg of the loop. The result was that the pressure pulse moved through the loop. This had little effect on the upper transition region Ov $629 \AA$ line, but the simulation produced a blinker-like curve in the lower transition region $\mathrm{C}_{\text {II }} 1037 \AA$ line.

For the analysis presented in this paper it was difficult to estimate the validity of any of the five theoretical blinker mechanisms suggested by Priest et al. (2002) and the suggestion of Harrison et al. (1999) that blinkers are triggered by an increase either in density or in brightness filling factor and not caused by a temperature increase.

\section{Conclusions and summary}

We presented the analyses of five time-series spectra of the quiet Sun of transition region lines Ov $629 \AA$, O vi $1032 \AA$ and $\mathrm{O}$ VI $1038 \AA$. The analyses detected 1086 blinkers and 778 explosive events. Somewhat more than a half of all detected explosive events took place during the course of a blinker in the same pixel. Cases were also found in which more than one explosive event occurred during a blinker. Due to this cospatiality and co-temporality of blinkers and explosive events one could think of a close relationship between blinkers and explosive events. However, analyses of blinker maximum intensity enhancements showed very different results for events with and without explosive events, and there are many explosive events with no associated blinker (the "classical" explosive event). These results suggest that the relationship is not so intimate; we would not expect such variations given a simple blinker, explosive event relationship suggested by many.

We also confirmed previous results obtained with CDS that blinker intensity light curves are time-structured as simple and complex ones. The average of their occurrences over five datasets showed that these two classes were equally represented. The analysis of the line profile parameters for these two classes and their correlations did not give any result which could further distinguish between simple and complex blinkers. During blinkers the intensity peaks mostly at the middle of their durations, while the line width peaks somewhat earlier than the intensity. This was a general characteristic of both simple and complex blinkers, as well as of all explosive events.

Based on the statistical analysis we concluded that explosive events and blinkers are independent phenomena which have to be explained separately. It is widely accepted that explosive events are bi-directional jets following a reconnection event. Although several proposals are offerred to explain the nature of blinkers the final word on this subject is not yet said, so that further blinker studies are needed. One certain direction in these investigations is in better understanding of blinkers relationship to the chromosphere. This can be done through the investigation of blinkers at different temperatures together with the corresponding underlying magnetic field. Further, it is necessary to continue improving various blinker models and compare them to observations.

Acknowledgements. We would like to thank R. Hammer for fruitful discussions and careful reading of the paper. We sincerely thank the referee R. A. Harrison for carefully reading and thoroughly commenting on the manuscript.We are grateful to the SUMER team and its open data policy. The SUMER project is financially supported by DLR, CNES, NASA and the ESA PRODEX programme (Swiss contribution). SOHO is a mission of international cooperation between ESA and NASA. The work of AB was supported by the Deutsche Forschungsgemeinschaft grant No. PE 782, which is gratefully acknowledged.

\section{References}

Arnaud, M., \& Rothenflug, R. 1985, A\&AS, 60, 425

Bewsher, D., Parnell, C. E., \& Harrison, R. A. 2002, Sol. Phys., 206, 21

Brković, A., Solanki, S. K., \& Rüedi, I. 2001, A\&A, 373, 1056

Brković, A., \& Peter, H. 2003, A\&A, 403, 287

Brueckner, G. E., \& Bartoe, J.-D. F. 1983, ApJ, 272, 329

Chae, J., Wang, H., Goode, P. R., et al. 2000, ApJ, 528, L119

Charbonneau, P. 1995, ApJS, 101, 309

Dere, K. P., Bartoe, J.-D. F., \& Brueckner, G. E. 1984, ApJ, 281, 870

Fontenla, J. M., Avrett, E. H., \& Loeser, R. 1993, ApJ, 406, 319

Handy, B. N., Acton, L. W., Kankelborg, C. C., et al. 1999, Sol. Phys., 187,229

Harrison, R. A., Sawyer, E. C., Carter, M. K., et al. 1995, Sol. Phys., 162,233

Harrison, R. A. 1997, Sol. Phys., 175, 467

Harrison, R. A., Lang, J., Brooks, D. H., \& Innes, D. E. 1999, A\&A, 351,1115

Harrison, R. A., Harra, L. K., Brković, A., \& Parnell, C. E. 2003, A\&A, 409, 755

Innes, D. E. 2001, A\&A, 378, 1067

Innes, D. E., Inhester, B., Axford, W. I., \& Wilhelm, K. 1997, Nature, 386,811

Innes, D. E., \& Tóth, G. 1999, Sol. Phys., 185, 127

Lemaire, P., Wilhelm, K., Curdt, W., et al. 1997, Sol. Phys., 170, 105

Madjarska, M. S., \& Doyle, J. G. 2002, A\&A, 382, 319

Marik, D., \& Erdélyi, R. 2002, A\&A, 393, L73

Parnell, C. E., Bewsher, D., \& Harrison, R. A. 2002, Sol. Phys., 206, 249

Peter, H., \& Judge, P. 1999, ApJ, 522, 1148

Peter, H., \& Brković, A. 2003, A\&A, 403, 287

Peter, H., Müller, D., \& Hansteen, V. 2004, in preparation

Priest, E. R., Hood, A. W., \& Bewsher, D. 2002, Sol. Phys., 205, 249

Teriaca, L., Madjarska, M. S., \& Doyle, J. G. 2002, A\&A, 392, 309

Wilhelm, K., Curdt, W., Marsch, E., et al. 1995, Sol. Phys., 162, 189

Winebarger, A. R., Updike, A. C., \& Reeves, K. K. 2002, ApJ, 570 L105 